\title{
28 Research Square \\ The Association Between Obstructive Sleep Apnea and Lung Nodule, Carcinoembryonic Antigen
}

\section{Ai-Ming Zeng}

the First Affiliated Hospital of Fujian Medical University

\section{Li-Da Chen}

Zhangzhou Affiliated Hospital of Fujian Medical University

\section{Zhi-Wei Zhao}

Zhangzhou Affiliated Hospital of Fujian Medical University

\section{Meng-Xue Chen}

the First Affiliated Hospital of Fujian Medical University

\section{Jie-Feng Huang}

the First Affiliated Hospital of Fujian Medical University

\section{Guo-Xiang Lai}

Fuzhou General Hospital of Fujian Medical University, Dongfang Hospital of Xiamen University, 900th Hospital of the Joint Logistics Support Force

Qi-Chang Lin ( $\square$ chang4e@126.com )

the First Affiliated Hospital of Fujian Medical University

\section{Research Article}

Keywords: Obstructive sleep apnea (OSA), lung nodules, carcinoembryonic antigen, lung cancer

Posted Date: April 15th, 2021

DOl: https://doi.org/10.21203/rs.3.rs-403766/v1

License: (c) (1) This work is licensed under a Creative Commons Attribution 4.0 International License.

Read Full License 


\section{Abstract}

Purpose: The association between obstructive sleep apnea (OSA) and cancer risks gaining more and more attention. Data on the association between OSA and lung cancer risk are limited. This study is to investigate whether a link exists between Low-dose computed tomography (LDCT) scanning of the chest findings, carcinoembryonic antigen (CEA) and OSA in patients suspected of OSA.

Methods: The cross-sectional study included patients aged 18 years or older who underwent continuous nocturnal polysomnography at our sleep center between February 2019 and November 2020. All subjects underwent chest LDCT and CEA. Patients with an apnea-hypopnea index(AHI) of $\geq 15 / \mathrm{h}$ were classified as clinically significant OSA group, whereas patients with an AHI $\otimes 15 / h$ were classified as control group.

Results: A total of 277 patients were enrolled in the study. 176 patients were categorized into the OSA group, while 101 patients were categorized into the control group. There is no relationship between any OSA-related parameter and presence of lung nodule or presence of $\geq 6 \mathrm{~mm}$ lung nodule in the binary logistic regression analysis. OSA group demonstrated a significant higher value of CEA than control group. Stepwise multiple linear regression analysis showed that lowest $\mathrm{O}_{2}$ saturation $(\beta=-0.256, p \llbracket 0.001)$, smoking status $(\beta=0.156, p=0.007)$ and age $(\beta=0.153, p=0.008)$ were independent predictors of elevated CEA.

Conclusion: In a group of patients suspected of OSA, we confirmed an independent correlation between nocturnal hypoxia and elevated CEA levels. OSA was not related with presence of pulmonary nodule or $\geq 6$ mm pulmonary nodule in LDCT.

\section{Introduction}

Obstructive sleep apnea (OSA), the most common form of sleep-disordered breathing (SDB), is mainly characterized by recurrent episodes of partial or complete upper airway obstructions during sleep that lead to intermittent hypoxia and sleep fragmentation. OSA is known to exert pathophysiologic and molecular disturbances on multiple organs and is linked to a variety of poor health outcomes. Recent evidences suggested that OSA was associated with increased incidence and mortality of cancer, especially in melanoma, lung cancer, prostate cancer, and colorectal cancer[1-4]. However, some studies opposed the results and indicated that OSA was not independently associated with cancer incidence and mortality[5]. It is not surprising that they commonly coexist in an individual as both OSA and cancer are common problems.

Lung cancer is the most commonly diagnosed cancer ( $11.6 \%$ of the total cases) and is the leading cause of cancer-related deaths ( $18.4 \%$ of the total cancer deaths) worldwide[6].It is well known that tobacco smoking is a major risk factor for the development of lung cancer. There are several studies examining the association between OSA and risk of lung cancer and its outcomes[7-10]. A study evaluating the prevalence of OSA in a lung cancer screening program found that OSA was very common in the high-risk population. Further multivariate analysis adjusted for confounders indicated that nocturnal hypoxemia 
was associated with positive screening findings (nodules $>6 \mathrm{~mm}$ )[11]. Another study investigating the prevalence of OSA in patients with newly-diagnosed lung cancer showed a high prevalence of OSA $(49 \%)$ in this population[12]. Nevertheless, the data of this issue are limited and the results remain inclusive. Furthermore, all studies focus on population with lung cancer or high risk of lung cancer. To our knowledge, there is no published study exploring this relationship based on population with OSA or clinical suspicion of OSA.

Low-dose computed tomography (LDCT) of the chest has been extensively used to screen lung cancer and shown to be efficacious at reducing mortality from lung cancer[13]. Serum tumor biomarkers can be accessed easily and economically, so they have been used as diagnostic adjunct and monitoring the course of lung cancer in the clinical setting. The National Academy of Clinical Biochemistry (NACB) recommends the serum CEA as a tumor biomarker for adjuvant lung cancer diagnosis. Hence, the present study aimed to explore the relationship between OSA and LDCT scanning of the chest finding since a cohort of patients studied for OSA suspicion. Furthermore, the association between OSA and CEA was also investigated in this study.

\section{Materials And Methods}

\section{Subjects}

Consecutive patients with age $>18$ years undergoing overnight polysomnography because of symptoms of sleep apnea between February 2019 and November 2020 in our sleep center were included in the study. The exclusion criteria included those patients with known diagnosis of OSA and receiving any OSArelated therapies, patients with known diagnosis of any malignancy and receiving any tumor-related therapies, patients with serious comorbidities, such as acute myocardial infarction. Pregnancy was also excluded. The informed consents were signed by all parents. This study was reviewed and approved by ethics committee of the First Affiliated Hospital of Fujian Medical University.

\section{Anthropometric measurements and polysomnography}

A detailed baseline evaluation including age, smoking habits, alcohol consumption, snoring, witnessed apneas, other medical history, and medication use were performed. Excessive daytime sleepiness was evaluated by the Epworth Sleepiness Scale. Body weight and height were measured in bare feet and light clothing in the morning by the same person using the same equipment. Body mass index (BMI) was calculated by dividing body weight by height squared $\left(\mathrm{kg} / \mathrm{m}^{2}\right)$. Waist circumference (WC) was measured at the level midway between the lower costal margin and the iliac crest. Neck circumference (NC) was measured at the level of the laryngeal prominence. A full-night polysomnography (P Series Sleep System, Compumedics, Melbourne, Australia) was performed for every participant at the sleep center. All of the PSG data were visually scored by trained technicians according to standardized criteria[14].Details have been described in our previous study[15]. The existing literature shows that there is a significant dose- 
response effect between $\mathrm{AHI}$ and the risk of cancer death, therefore patients with an apnea-hypopnea index (AHI) higher than or equal to 15 were categorized as having clinically significant OSA. Patients with an $\mathrm{AHI}$ less than 15 were categorized into control group[16, 17].

\section{Blood samples}

A fasting blood sample was drawn after a full night sleep study and then centrifuged at $3000 \mathrm{rpm}$ for 10 min. CEA was quantitatively measured using electrochemiluminescence immunoassay (ECLIA) assay kits, according to the manufacturer's instructions (Cobas, Roche Diagnostics, Germany).

\section{LDCT of the chest}

All subjects were examined using a multilayer helical scanner at a low-dose project (105 mA, 120 kV,2 mm overlapping sections). LDCT examination was performed in a single breath hold during complete inhalation. Chest CT image uploaded to Picture Archiving and Communication Systems (PACS). An experienced radiologist distinguished and described pulmonary nodules based on their size, presence, and type (solid, nonsolid, or partially solid) and the location of the nodules. It was reviewed by another senior radiologist. Pulmonary nodules was represented by the average the maximum nodule diameter and the nodule diameter perpendicular to this maximum.

\section{Statistical analysis}

The SPSS 19.0 package (SPSS Inc, Chicago, IL, USA) was used for the statistical analysis. GraphPad Prism 8 (GraphPad Software, USA) was used to draw the graphs. Normal distribution tests were performed on the data prior to analysis. In the case of normal distribution, the Student's t test was used to compare the means between two groups, or in the case of non-normal distribution, the Mann-Whitney $U$ test was used for non-parametric data. Where appropriate, the categorical variables were compared using chi-square test or Fisher exact test. Binary logistic regression was used to analyze the relationship between OSA-related parameters and presence of pulmonary nodule, $\geq 6 \mathrm{~mm}$ pulmonary nodule. Correlations between variables were explored using the Pearson correlation test. Multiple linear regression was used to determine the independent predictors of CEA. When p-value was less than 0.05 , it was considered statistically significant.

\section{Results}

\section{Comparison of variables of all patients}

The study group comprised 277 patients with 206 male and 71 female. The mean age was $53.49 \pm 12.71$ years, mean BMI was $27.34 \pm 4.77 \mathrm{~kg} / \mathrm{m}^{2}$, mean AHI was $28.89 \pm 21.82$. 101 patients with $A H \mathrm{l}<15$ were categorized into control group, the remaining 176 patients with $\mathrm{AHI} \geq 15$ were categorized into OSA 
group. Table 1 shows the comparison of baseline variables of OSA and controls. There were no significant differences in age, prevalence of diabetes, COPD, emphysema, smoking status, alcohol consumption between groups. The male sex ratio, prevalence of hypertension, dyslipidemia, BMI, NC, WC, $\mathrm{AHI}$, oxygen desaturation index (ODI), the percentage of sleep time with $\mathrm{SpO}_{2}(\mathrm{~T} 90 \%)$, epworth sleepiness scale (ESS) score of OSA group was significantly higher than control group. The OSA patients had significantly lower $\mathrm{LaSO}_{2}$, average $\mathrm{SpO}_{2}$ compared with patients in control group. No significant differences were observed in presence of lung nodule and presence of lung nodule $\geq 6 \mathrm{~mm}$ between groups. 
Table 1

Comparison of variables of OSA and controls

\begin{tabular}{|c|c|c|c|}
\hline & Controls & OSA & $p$-values \\
\hline Subjects $n$ & 101 & 176 & \\
\hline Age, years & $52.57 \pm 14.10$ & $54.01 \pm 11.85$ & 0.389 \\
\hline Male sex, number(\%) & $67(66.3)$ & 139(79.0) & 0.020 \\
\hline Hypertension, number(\%) & $38(37.6)$ & 108(61.4) & $<0.001$ \\
\hline Diabetes, number(\%) & 19(18.8) & $33(18.8)$ & 0.990 \\
\hline Dyslipidemia, number(\%) & $6(5.9)$ & $30(17.0)$ & 0.008 \\
\hline COPD, number(\%) & $4(4.0)$ & $4(2.3)$ & 0.420 \\
\hline Emphysema, number(\%) & $2(2.0)$ & $2(1.1)$ & 0.624 \\
\hline \multicolumn{4}{|l|}{ Tobacco status } \\
\hline Never, number(\%) & $69(68.3)$ & $102(58.0)$ & \\
\hline Former, number(\%) & 14(13.9) & 23(13.1) & \\
\hline Active, number(\%) & 18(17.8) & $51(29.0)$ & 0.113 \\
\hline Alcohol consumption, number (\%) & 24(23.8) & $60(34.1)$ & 0.072 \\
\hline $\operatorname{BMI}\left(\mathrm{kg} / \mathrm{m}^{2}\right)$ & $25.67 \pm 4.23$ & $28.29 \pm 4.80$ & $<0.001$ \\
\hline $\mathrm{NC}(\mathrm{cm})$ & $37.30 \pm 3.20$ & $39.75 \pm 3.77$ & $<0.001$ \\
\hline $\mathrm{WC}(\mathrm{cm})$ & $93.75 \pm 12.25$ & $101.31 \pm 11.80$ & $<0.001$ \\
\hline $\mathrm{AHI}$ & $8.14(4.80-11.20)$ & $37.70(26.40-51.05)$ & $<0.001$ \\
\hline ODI & $5.70(2.80-8.20)$ & $28.60(18.38-46.70)$ & $<0.001$ \\
\hline T90\% (\%) & $0.14(0.02-0.47)$ & $3.37(0.74-10.76)$ & $<0.001$ \\
\hline $\mathrm{LaSO}_{2}(\%)$ & $85.00(82.00-88.00)$ & $76.50(65.00-82.00)$ & $<0.001$ \\
\hline Average $\mathrm{SpO}_{2}(\%)$ & $95.00(94.00-96.00)$ & $93.00(91.00-94.00)$ & $<0.001$ \\
\hline
\end{tabular}

Normally distributed data were expressed as mean \pm SD, skewed data (including AHI, ODI, T90\%, $\mathrm{LaSO}_{2}$, Average $\mathrm{SaO}_{2}$ ) were presented as median (interquartile range). Categorical variables were expressed as number (percentage).

Abbreviation: OSA = obstructive sleep apnea, $\mathrm{COPD}=$ chronic obstructive pulmonary diseases, $\mathrm{BMI}=$ body mass index, $\mathrm{NC}=$ neck circumference, $\mathrm{WC}$ = waist circumference, $\mathrm{AHI}=$ apnea-hypopnea index, $\mathrm{ODI}=$ oxygen desaturation index, $\mathrm{T} 90 \%$ =the percentage of sleep time with $\mathrm{SpO}_{2}$, $\mathrm{LaSO}_{2}=$ lowest $\mathrm{O}_{2}$ saturation, ESS = epworth sleepiness scale. 


\begin{tabular}{|c|c|c|c|}
\hline & Controls & OSA & $p$-values \\
\hline ESS score & $5.92 \pm 4.48$ & $8.65 \pm 5.89$ & $<0.001$ \\
\hline Nodule, number (\%) & $51(50.5)$ & $92(52.3)$ & 0.776 \\
\hline Nodules $\geq 6 \mathrm{~mm}$, number (\%) & 19(18.8) & 21(11.9) & 0.117 \\
\hline \multicolumn{4}{|c|}{$\begin{array}{l}\text { Normally distributed data were expressed as mean } \pm \mathrm{SD} \text {, skewed data (including } \mathrm{AHI}, \mathrm{ODI}, \mathrm{T} 90 \% \text {, } \\
\mathrm{LaSO}_{2} \text {, Average } \mathrm{SaO}_{2} \text { ) were presented as median (interquartile range). Categorical variables were } \\
\text { expressed as number (percentage). }\end{array}$} \\
\hline \multicolumn{4}{|c|}{$\begin{array}{l}\text { Abbreviation: OSA = obstructive sleep apnea, } \mathrm{COPD}=\text { chronic obstructive pulmonary diseases, } \mathrm{BMI}= \\
\text { body mass index, } \mathrm{NC}=\text { neck circumference, } \mathrm{WC}=\text { waist circumference, } \mathrm{AHI}=\text { apnea-hypopnea index, } \\
\mathrm{ODI}=\text { oxygen desaturation index, } \mathrm{T} 90 \%=\text { the percentage of sleep time with } \mathrm{SpO}_{2}, \mathrm{LaSO}_{2}=\text { lowest } \mathrm{O}_{2} \\
\text { saturation, ESS = epworth sleepiness scale. }\end{array}$} \\
\hline
\end{tabular}

\section{Comparison of variables of patients with lung nodules}

Out of 277 patients included, 143 patients with lung nodules were further analyzed. They were also divided into control group and OSA group. The detailed information is shown in the Table 2. Similar to the results yielded from whole population, the prevalence of hypertension, dyslipidemia, BMI, NC, WC, sleep parameters and ESS score were significantly different between OSA and control group. There was no statistical difference in presence of lung nodule and presence of lung nodule $\geq 6 \mathrm{~mm}$ between groups. 
Table 2

Comparison of variables of OSA and controls in patients with lung nodules

\begin{tabular}{|c|c|c|c|}
\hline & Controls & OSA & $p$-values \\
\hline Subjects n & 51 & 92 & \\
\hline Age, years & $5.24 \pm 14.50$ & $55.51 \pm 12.36$ & 0.905 \\
\hline Male sex, number(\%) & $32(62.7)$ & $69(75.0)$ & 0.123 \\
\hline Hypertension, number(\%) & $14(27.5)$ & $59(64.1)$ & 0.000 \\
\hline Diabetes, number(\%) & $8(15.7)$ & $21(22.8)$ & 0.309 \\
\hline Dyslipidemia, number(\%) & $1(2.0)$ & $14(15.2)$ & 0.013 \\
\hline COPD, number(\%) & $3(5.9)$ & $2(2.2)$ & 0.348 \\
\hline Emphysema, number(\%) & $0(0.0)$ & $1(1.1)$ & 1.000 \\
\hline \multicolumn{4}{|l|}{ Tobacco status } \\
\hline Never, number(\%) & $33(64.7)$ & $56(60.9)$ & \\
\hline Former, number(\%) & $9(17.6)$ & $13(14.1)$ & \\
\hline Active, number(\%) & $9(17.6)$ & $23(25.0)$ & 0.567 \\
\hline Alcohol consumption, number (\%) & 17(33.3) & $28(30.4)$ & 0.721 \\
\hline $\mathrm{BMI}\left(\mathrm{kg} / \mathrm{m}^{2}\right)$ & $25.33 \pm 4.50$ & $28.17 \pm 5.06$ & 0.001 \\
\hline $\mathrm{NC}(\mathrm{cm})$ & $37.01 \pm 3.23$ & $39.63 \pm 3.78$ & $<0.001$ \\
\hline $\mathrm{WC}(\mathrm{cm})$ & $94.53 \pm 11.52$ & $101.26 \pm 13.00$ & 0.020 \\
\hline $\mathrm{AHI}$ & $8.90(6.80-11.80)$ & $36.60(26.25-48.35)$ & $<0.001$ \\
\hline ODI & $5.80(2.70-8.90)$ & $27.80(18.93-37.68)$ & $<0.001$ \\
\hline T90\% (\%) & $0.13(0.02-0.46)$ & $1.88(0.44-8.56)$ & $<0.001$ \\
\hline ESS score & $5.45 \pm 4.47$ & $8.20 \pm 6.17$ & 0.003 \\
\hline MPN & $24(47.1)$ & $50(54.3)$ & 0.403 \\
\hline GGO & $6(11.8)$ & $12(13.0)$ & 0.825 \\
\hline
\end{tabular}

Normally distributed data were expressed as mean \pm SD, skewed data (including AHI, ODI, T90\%) were presented as median (interquartile range). Categorical variables were expressed as number (percentage).

Abbreviation: OSA = obstructive sleep apnea, $\mathrm{COPD}=$ chronic obstructive pulmonary diseases, $\mathrm{BMI}=$ body mass index, $\mathrm{NC}=$ neck circumference, $\mathrm{WC}$ = waist circumference, $\mathrm{AHI}=$ apnea-hypopnea index $\mathrm{ODI}=$ oxygen desaturation index, $\mathrm{T} 90 \%=$ the percentage of sleep time with $\mathrm{SpO}_{2}$, ESS = epworth sleepiness scale, MPN = multiple pulmonary nodules, GGO = ground-glass opacity . 


\begin{tabular}{|lccc|}
\hline & Controls & OSA & $p$-values \\
\hline Nodule size & $0.59 \pm 0.59$ & $0.46 \pm 0.30$ & 0.075 \\
\hline Nodules $\geq 6 \mathrm{~mm}$ & $19(37.3)$ & $21(22.8)$ & 0.066 \\
\hline $\begin{array}{l}\text { Normally distributed data were expressed as mean } \pm \mathrm{SD}, \text { skewed data (including AHI, ODI, T90\%) were } \\
\text { presented as median (interquartile range). Categorical variables were expressed as number } \\
\text { (percentage). }\end{array}$ & & \\
\hline $\begin{array}{l}\text { Abbreviation: OSA = obstructive sleep apnea, COPD = chronic obstructive pulmonary diseases, } \mathrm{BMI}= \\
\text { body mass index, NC = neck circumference, WC = waist circumference, AHI = apnea-hypopnea index, } \\
\text { ODI = oxygen desaturation index, T90\%=the percentage of sleep time with } \mathrm{SpO}_{2}, \text { ESS = epworth } \\
\text { sleepiness scale, MPN = multiple pulmonary nodules, GGO = ground-glass opacity. }\end{array}$ \\
\hline
\end{tabular}

\section{Relationship between OSA and lung nodules}

Binary logistic regression was further performed to analyze the relationship between OSA-related parameters and presence of lung nodule, $\geq 6 \mathrm{~mm}$ lung nodule. The results are presented in the Table 3 . There is no relationship between any OSA-related parameter and presence of lung nodule or presence of $\geq 6 \mathrm{~mm}$ lung nodule. The correlation analysis indicated that the size of lung nodule was not correlated with any OSA-related parameter (Fig. 1).

Table 3

Univariate analysis for lung nodules

\begin{tabular}{|lllllll|}
\hline & \multicolumn{3}{c|}{ Presence of lung nodules } & \multicolumn{4}{c|}{ Presence of $\geq 6 \mathrm{~mm}$ lung nodules } \\
\hline Parameter & $\mathrm{OR}$ & $95 \% \mathrm{Cl}$ & $p$-values & $\mathrm{OR}$ & $95 \% \mathrm{Cl}$ & $p$-values \\
\hline $\mathrm{AHI}$ & 0.996 & $0.985-1.006$ & 0.417 & 0.987 & $0.970-1.004$ & 0.135 \\
\hline $\mathrm{ODI}$ & 0.993 & $0.982-1.004$ & 0.191 & 0.986 & $0.969-1.004$ & 0.131 \\
\hline $\mathrm{T} 90 \%(\%)$ & 0.981 & $0.960-1.002$ & 0.074 & 0.969 & $0.927-1.013$ & 0.160 \\
\hline $\mathrm{LaSO}_{2}(\%)$ & 1.007 & $0.988-1.026$ & 0.492 & 1.014 & $0.984-1.045$ & 0.353 \\
\hline Average $\mathrm{SpO}_{2}(\%)$ & 1.002 & $0.938-1.070$ & 0.957 & 1.004 & $0.913-1.103$ & 0.941 \\
\hline $\begin{array}{l}\text { Abbreviation: } \mathrm{AHI}=\text { apnea-hypopnea index, ODI = oxygen desaturation index, T90\%=the percentage of } \\
\text { sleep time with } \mathrm{SpO}_{2} \text {, LaSO2 = lowest } \mathrm{O}_{2} \text { saturation, OR = odds ratio, Cl = confidence interval. }\end{array}$ \\
\hline
\end{tabular}

\section{Relationship between OSA and CEA}


Figure 2 showed the comparison of CEA between OSA and control group. OSA group demonstrated a significant higher value of CEA than control group $(2.12 \pm 1.15 \mathrm{vs} .2 .53 \pm 1.66 \mathrm{ng} / \mathrm{ml})$. The correlation analysis revealed that age, the presence of diabetes, tobacco status, $\mathrm{BMI}, \mathrm{WC}, \mathrm{AHI}, \mathrm{ODI}, \mathrm{T} 90 \%, \mathrm{LaSO}_{2}$, average $\mathrm{SpO}_{2}$ were the factors correlated significantly with CEA (Table 4). Stepwise multiple linear regression was performed to determine the independent variables of CEA. Age, sex ratio, presence of hypertension, diabetes, dyslipidemia, COPD, tobacco status, alcohol consumption, BMI, WC, AHI, ODI, $\mathrm{T} 90 \%, \mathrm{LaSO}_{2}$, and average $\mathrm{SpO}_{2}$ were taken as independent variables entering into the regression model. The results showed that $\mathrm{LaSO}_{2}(\beta=-0.256, p<0.001)$, tobacco status $(\beta=0.156, p=0.007)$ and age $(\beta=$ $0.153, p=0.008$ ) were independent predictors of elevated CEA. 
Table 4

Pearson correlation coefficients between CEA and demographic data, sleep data.-

\begin{tabular}{|c|c|c|}
\hline & $\operatorname{CEA}(n=$ & \\
\hline & $r$ & $p$-values \\
\hline Age & 0.148 & 0.014 \\
\hline Sex & -0.083 & 0.168 \\
\hline Hypertension, number(\%) & 0.074 & 0.217 \\
\hline Diabetes, number(\%) & 0.130 & 0.030 \\
\hline Dyslipidemia, number(\%) & 0.044 & 0.465 \\
\hline COPD, number(\%) & -0.052 & 0.393 \\
\hline Tobacco status & 0.175 & 0.003 \\
\hline Alcohol consumption & -0.012 & 0.838 \\
\hline $\operatorname{BMI}\left(\mathrm{kg} / \mathrm{m}^{2}\right)$ & 0.134 & 0.026 \\
\hline WC(cm) & 0.146 & 0.015 \\
\hline $\mathrm{AHI}$ & 0.159 & 0.008 \\
\hline ODI & 0.138 & 0.021 \\
\hline T90\% (\%) & 0.220 & $<0.001$ \\
\hline $\mathrm{LaSO}_{2}(\%)$ & -0.275 & $<0.001$ \\
\hline Average $\mathrm{SpO}_{2}(\%)$ & -0.176 & 0.003 \\
\hline ESS score & -0.003 & 0.955 \\
\hline $\begin{array}{l}\text { Abbreviation: } C E A=\text { carcin } \\
\text { = body mass index, } W C=\checkmark \\
\text { desaturation index, } \mathrm{T} 90 \%= \\
=\text { epworth sleepiness scal }\end{array}$ & $\begin{array}{l}\text { honic ob } \\
\text { a-hypop } \\
\text { ith } \mathrm{SpO}_{2}\end{array}$ & $\begin{array}{l}\text { Almonary diseases, BMI } \\
\mathrm{DI}=\text { oxygen } \\
\text { west } \mathrm{O}_{2} \text { saturation, ESS }\end{array}$ \\
\hline
\end{tabular}

\section{Discussion}

In a cohort of patients with suspicion of OSA, we demonstrated that nocturnal hypoxia was independently related to the elevated of serum CEA level after adjusting confounding factors. OSA was not related with presence of pulmonary nodule or $\geq 6 \mathrm{~mm}$ pulmonary nodule in LDCT.

There is growing interest in evaluating the association between OSA and cancer incidence. Several large cohort studies have investigated whether OSA increased the risk of cancer. A community-based cohort study with long term follow up found that moderate-severe OSA was significantly associated with 
incident cancer after adjusting confounding factors[18]. A multicenter, clinical cohort study including 4,910 patients investigated for suspected OSA revealed that severe OSA was associated with higher incidence of cancer, especially inpatients aged $<65$ years and men[2]. However, some study did not support an independent relationship between OSA and cancer incidence. Over a 7.8-year follow-up of 10,149 patients, Kendzerska et al[1] found that the severity of sleep apnea was not significantly associated with either prevalent or incident cancer after adjustment for age, sex, BMI and smoking status at baseline. A recent prospective study with 8-year follow-up of 65,330 older women showed that OSA was associated with a $15 \%$ increase in total cancer risk. The association became non-significant after adjustment for multiple cancer risk factors[19].

Lung cancer is the most commonly seen cancer. Some studies focused on the relationship between OSA and risk of lung cancer and its mortality. A study performed in Germany reported high prevalence of OSA in newly diagnosed lung cancer patients, with the incidence of moderate to severe OSA reaching $17 \%$. But it could not determine whether OSA affects the prevalence of lung cancer[12]. Another study performed in Spain reported that the prevalence of OSA was $80 \%$ in consecutive patients with newly diagnosed lung cancer[9]. A cross-sectional study including 302 patients for investigating relationship between OSA and lung cancer discovered that $\mathrm{AHI}$ and nocturnal hypoxemia did increase the incidence of lung cancer, especially moderate and severe OSA[7]. However, data is still limited in this issue and the conclusion is controversial.

Serum tumor markers are becoming more and more important in the diagnosis and staging of malignant tumors. CEA is one of the most commonly used tumor markers. Its overexpression is first found in colorectal cancer, and then in respiratory tract and other organs. Although the use of CEA in patients with lung cancer is still controversial, its low cost and easy availability make it a relatively reliable indicator of lung cancer. CEA is essentially a marker of adenocarcinoma, while it is closely related to TNM stage and tumor aggressiveness, so it has become an indicator of lung adenocarcinoma prognosis.

The mechanisms likely to be involved in the association between OSA and cancer included systemic inflammation, oxidative stress, angiogenesis, immune dysfunction. In an animal study, Almendros et al[20] demonstrated that mice exposed to intermittent-hypoxia(IH)exhibited accelerated tumor growth and presented more tumor invasion. Tumor-associated macrophages from IH-exposed mice significantly increased TC1 migration and extravasation compared with normoxia group. Another animal study also confirmed that $\mathrm{IH}$ markedly enhanced the number and weight of lung metastatic colonies in mouse. Compared with control group, Lung tissues with tumor metastasis from IH group showed increased oxidative stress, as assessed by p22phox and superoxide dismutase (SOD) mRNA levels and the NF-E2related factor 2 (NRF2) protein Level, and inflammation, as assessed by Tumor Necrosis Factor - $a$ (TNFa) and interleukin - 6 mRNA levels and the NF-KB P65 protein level[21]. Zhang et al[22] showed that micro-vessel density (MVD) and vascular endothelial growth factor (VEGF) were significantly higher in tumor from IH animal than control group. Treatment with endostatin attenuated serum and tissue VEGF levels, lowering the MVD values. This study indicated that $\mathrm{IH}$ might accelerate tumor growth by facilitating the angiogenesis in tumor tissue. 
Our study revealed that OSA was not related with presence of pulmonary nodule or $\geq 6 \mathrm{~mm}$ pulmonary nodule in LDCT. Generally, patients with $\geq 6 \mathrm{~mm}$ pulmonary nodule were be considered to be at high risk of lung cancer, so we choose this standard. This is contradictory to the conclusion from a study, which indicated that nocturnal hypoxemia was associated with presence of $>6 \mathrm{~mm}$ lung nodules on multivariate analysis adjusted for confounders $(O R=2.6)[11]$. The differences in the results might due to the different patient group. We included a group of patients suspected for OSA. The study by PerezWarnisher et al[11] included a group of patients with age and smoking criteria fulfilling a high risk of lung cancer (age $55-75$ years; pack-years $>30$ ). We also found that nocturnal hypoxia was independently related to the elevated of serum CEA level after adjusting confounding factors. This is consistent with a previous study reporting that the AHI score and ODI score were positively associated with the CEA level in T2DM patients[23]. Serum CEA levels are not only increased in lung cancer but also increased in other types of cancer such as colorectal cancer. Actually, an previous epidemiological study showed that OSA was associated with an increased risk of colorectal cancer[24]. So it cannot be excluded that OSA was associated with other type of cancer (e.g., colorectal cancer).

However, it is vital to note the limitations of this study. First of all, this is a cross-sectional study, therefore the observed associations do not indicate cause-effect relationships. Secondly, the sample size of this study is relatively small compared to the sample size of the community-based cohort study. Thirdly, histological findings of lung nodules were not obtained in this study. Finally, serum CEA level can be affected by many diseases or factors. Other cancer type such as colorectal cancer might not be excluded due to lack of endoscopic or PET-CT examining.

In conclusion, OSA was independently related to the elevated of serum CEA level, but not with presence of pulmonary nodule or $\geq 6 \mathrm{~mm}$ pulmonary nodule in LDCT. Further well-designed longitudinal studies with pathology available are needed to identify the association between OSA and risk of lung cancer.

\section{Abbreviations}

OSA: obstructive sleep apnea, LDCT: Low-dose computed tomography, CEA: carcinoembryonic antigen, SDB: sleep-disordered breathing, COPD: chronic obstructive pulmonary diseases, BMI: body mass index, NC: neck circumference, WC: waist circumference, AHI: apnea-hypopnea index, ODI: oxygen desaturation index, $\mathrm{T} 90 \%$ : the percentage of sleep time with $\mathrm{SpO}_{2}, \mathrm{LaSO}_{2}$ : lowest $\mathrm{O}_{2}$ saturation, ESS: epworth sleepiness scale, MPN: multiple pulmonary nodules, GGO: ground-glass opacity, OR: odds ratio, $\mathrm{Cl}$ : confidence interval.

\section{Declarations}

\section{Acknowledgements}

Not applicable.

\section{Authors' contributions}


AMZ, LDC, ZWZ and QCL conceived and designed the study, analyzed the data and drafted this manuscript. MXC, JFH and GXL contributed to analysis of the data, and revising of the manuscript. All authors have read and approved the final manuscript.

\section{Funding}

This work was supported by grant from Science and Technology Major Project of Fujian Province (2017YZ0001-2)

\section{Availability of data and materials}

All data generated or analyzed during this study are included in this published article.

\section{Ethics approval and consent to participate}

The study protocol conformed to the 1975 Declaration of Helsinki and was reviewed and approved by ethics committee of the First Affiliated Hospital of Fujian Medical University. Written informed consent was obtained from each parent of participant.

\section{Consent for publication}

Not applicable.

\section{Competing interests}

The authors declare that they have no known competing financial interests or personal relationships that could have appeared to influence the work reported in this paper.

\section{References}

1. Kendzerska T, Leung RS, Hawker G, Tomlinson G, Gershon AS: Obstructive sleep apnea and the prevalence and incidence of cancer. CMAJ : Canadian Medical Association journal = journal de l'Association medicale canadienne 2014, 186(13):985-992.

2. Campos-Rodriguez F, Martinez-Garcia MA, Martinez M, Duran-Cantolla J, Pena Mde L, Masdeu MJ, Gonzalez M, Campo F, Gallego I, Marin JM et al: Association between obstructive sleep apnea and cancer incidence in a large multicenter Spanish cohort. American journal of respiratory and critical care medicine 2013, 187(1):99-105.

3. Nieto FJ, Peppard PE, Young T, Finn L, Hla KM, Farre R: Sleep-disordered breathing and cancer mortality: results from the Wisconsin Sleep Cohort Study. American journal of respiratory and critical care medicine 2012, 186(2):190-194.

4. Santamaria-Martos F, Benitez I, Giron C, Barbe F, Martinez-Garcia MA, Hernandez L, Montserrat JM, Nagore E, Martorell A, Campos-Rodriguez $\mathrm{F}$ et al: Biomarkers of carcinogenesis and tumour growth in 
patients with cutaneous melanoma and obstructive sleep apnoea. The European respiratory journal 2018, 51(3).

5. Zhang XB, Peng LH, Lyu Z, Jiang XT, Du YP: Obstructive sleep apnoea and the incidence and mortality of cancer: a meta-analysis. European journal of cancer care 2017, 26(2).

6. Bray F, Ferlay J, Soerjomataram I, Siegel RL, Torre LA, Jemal A: Global cancer statistics 2018: GLOBOCAN estimates of incidence and mortality worldwide for 36 cancers in 185 countries. CA: a cancer journal for clinicians 2018, 68(6):394-424.

7. Seijo LM, Perez-Warnisher MT, Giraldo-Cadavid LF, Oliveros H, Cabezas E, Troncoso MF, Gomez T, Melchor R, Pinillos EJ, El Hachem A et al: Obstructive sleep apnea and nocturnal hypoxemia are associated with an increased risk of lung cancer. Sleep medicine 2019, 63:41-45.

8. Huang HY, Lin SW, Chuang LP, Wang CL, Sun MH, Li HY, Chang CJ, Chang SC, Yang CT, Chen NH: Severe Obstructive Sleep Apnea Associated With Higher Risk of Mortality in Stage III and IV Lung Cancer. Journal of clinical sleep medicine: JCSM : official publication of the American Academy of Sleep Medicine 2020.

9. Cabezas E, Perez-Warnisher MT, Troncoso MF, Gomez T, Melchor R, Pinillos EJ, El Hachem A, Gotera C, Rodriguez P, Mahillo Fernandez I et al: Sleep Disordered Breathing Is Highly Prevalent in Patients with Lung Cancer: Results of the Sleep Apnea in Lung Cancer Study. Respiration; international review of thoracic diseases 2019, 97(2):119-124.

10. Liu Y, Lao M, Chen J, Lu M, Luo S, Ou Q, Luo Z, Yuan P, Chen J, Ye G et al: Short-term prognostic effects of circulating regulatory T-Cell suppressive function and vascular endothelial growth factor level in patients with non-small cell lung cancer and obstructive sleep apnea. Sleep medicine 2020, 70:88-96.

11. Perez-Warnisher MT, Cabezas E, Troncoso MF, Gomez T, Melchor R, Pinillos EJ, El Hachem A, Gotera $\mathrm{C}$, Rodriguez $\mathrm{P}$, Mahillo I et al: Sleep disordered breathing and nocturnal hypoxemia are very prevalent in a lung cancer screening population and may condition lung cancer screening findings: results of the prospective Sleep Apnea In Lung Cancer Screening (SAILS) study. Sleep medicine 2019, 54:181186.

12. Dreher M, Kruger S, Schulze-Olden S, Keszei A, Storre JH, Woehrle H, Arzt M, Muller T: Sleepdisordered breathing in patients with newly diagnosed lung cancer. BMC pulmonary medicine 2018, 18(1):72.

13. National Lung Screening Trial Research T, Aberle DR, Adams AM, Berg CD, Black WC, Clapp JD, Fagerstrom RM, Gareen IF, Gatsonis C, Marcus PM et al: Reduced lung-cancer mortality with low-dose computed tomographic screening. The New England journal of medicine 2011, 365(5):395-409.

14. Schulz H: Phasic or transient? Comment on the terminology of the AASM manual for the scoring of sleep and associated events. Journal of clinical sleep medicine : JCSM : official publication of the American Academy of Sleep Medicine 2007, 3(7):752.

15. Lin QC, Chen LD, Chen GP, Zhao JM, Chen X, Huang JF, Wu LH: Association between nocturnal hypoxia and liver injury in the setting of nonalcoholic fatty liver disease. Sleep \& breathing $=$ Schlaf \& 
Atmung 2015, 19(1):273-280.

16. Polotsky VY, Patil SP, Savransky V, Laffan A, Fonti S, Frame LA, Steele KE, Schweizter MA, Clark JM, Torbenson MS et al: Obstructive sleep apnea, insulin resistance, and steatohepatitis in severe obesity. American journal of respiratory and critical care medicine 2009, 179(3):228-234.

17. Ross SD, Sheinhait IA, Harrison KJ, Kvasz M, Connelly JE, Shea SA, Allen IE: Systematic review and meta-analysis of the literature regarding the diagnosis of sleep apnea. Sleep 2000, 23(4):519-532.

18. Marshall NS, Wong KK, Cullen SR, Knuiman MW, Grunstein RR: Sleep apnea and 20-year follow-up for all-cause mortality, stroke, and cancer incidence and mortality in the Busselton Health Study cohort. Journal of clinical sleep medicine : JCSM : official publication of the American Academy of Sleep Medicine 2014, 10(4):355-362.

19. Huang T, Lin BM, Stampfer MJ, Schernhammer ES, Saxena R, Tworoger SS, Redline S: Associations of Self-reported Obstructive Sleep Apnea with Total and Site-specific Cancer Risk in Older Women: A Prospective Study. Sleep 2020.

20. Almendros I, Wang Y, Becker L, Lennon FE, Zheng J, Coats BR, Schoenfelt KS, Carreras A, Hakim F, Zhang SX et al: Intermittent hypoxia-induced changes in tumor-associated macrophages and tumor malignancy in a mouse model of sleep apnea. American journal of respiratory and critical care medicine 2014, 189(5):593-601.

21. Li L, Ren F, Qi C, Xu L, Fang Y, Liang M, Feng J, Chen B, Ning W, Cao J: Intermittent hypoxia promotes melanoma lung metastasis via oxidative stress and inflammation responses in a mouse model of obstructive sleep apnea. Respiratory research 2018, 19(1):28.

22. Zhang XB, Yang YY, Zeng Y, Zeng HQ, Fu BB, Ko CY, Luo X, Du YP, Chen LD, Lai YT et al: Anti-tumor effect of endostatin in a sleep-apnea mouse model with tumor. Clinical \& translational oncology : official publication of the Federation of Spanish Oncology Societies and of the National Cancer Institute of Mexico 2018.

23. Zhou J, Huang X, Jiang X: Effects of Obstructive Sleep Apnea-Hypopnea Syndrome on Serum Carcinoembryonic Antigen Levels in Patients with Type 2 Diabetes Mellitus. Medical science monitor : international medical journal of experimental and clinical research 2019, 25:3558-3565.

24. Chen CY, Hu JM, Shen CJ, Chou YC, Tian YF, Chen YC, You SL, Hung CF, Lin TC, Hsiao CW et al: Increased incidence of colorectal cancer with obstructive sleep apnea: a nationwide populationbased cohort study. Sleep medicine 2020, 66:15-20.

\section{Figures}



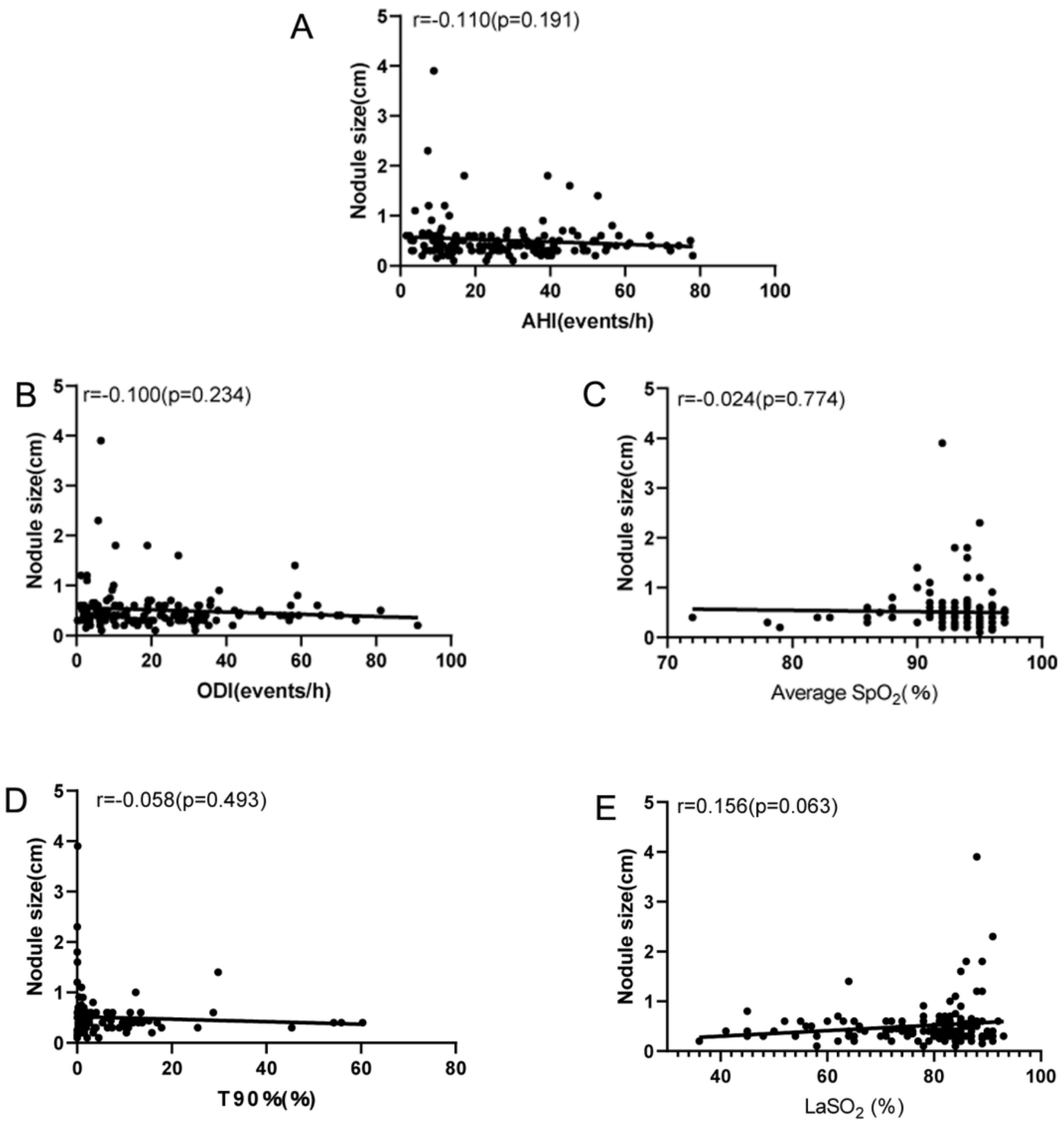

Figure 1

The correlation analysis OSA-related parameter and the size of lung nodule. Abbreviation: OSA=obstructive sleep apnea, $\mathrm{AHI}=$ apnea-hypopnea index, $\mathrm{ODI}=$ oxygen desaturation index, $\mathrm{T} 90 \%=$ the percentage of sleep time with $\mathrm{SpO} 2$, LaSO2=lowest 02 saturation 


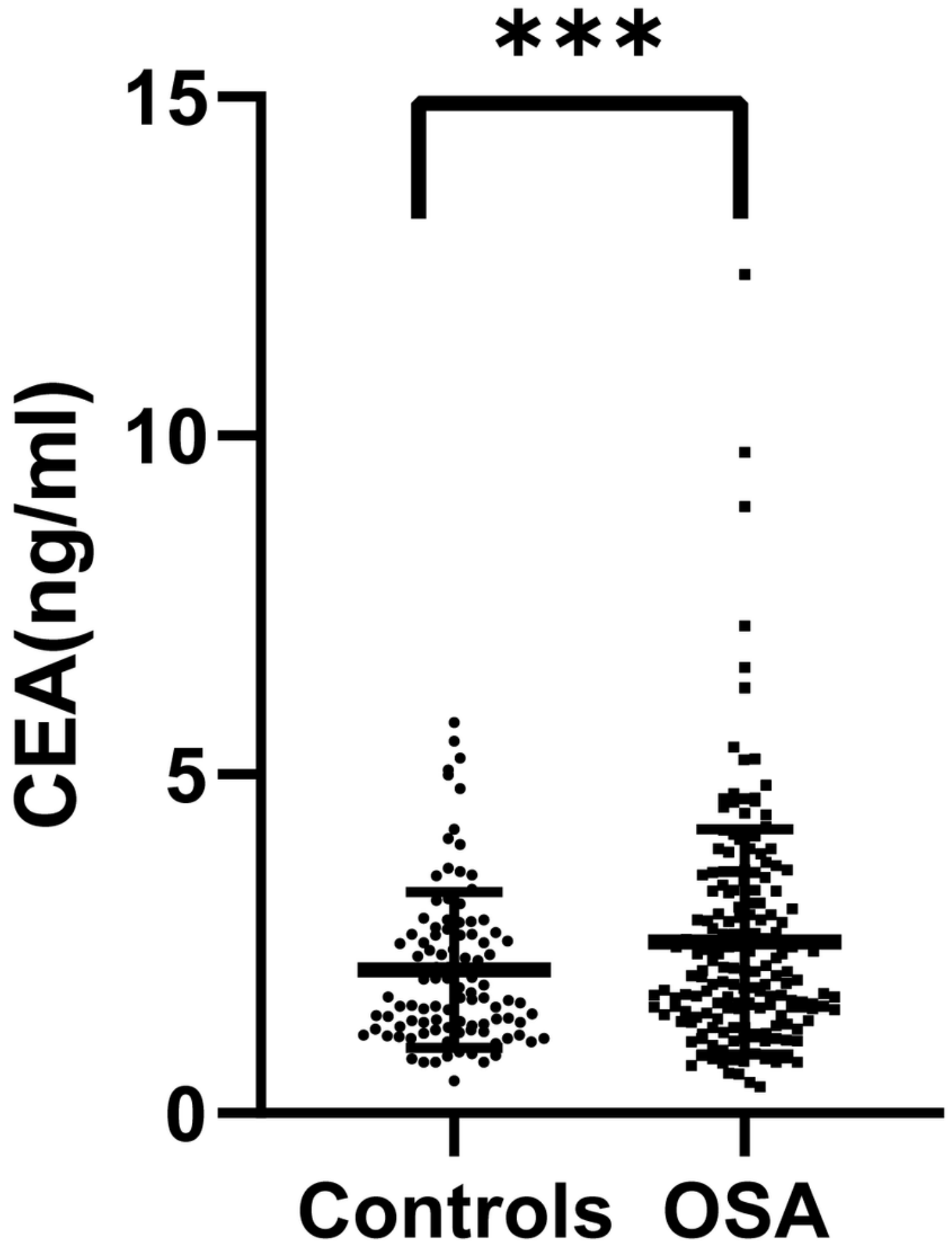

Figure 2

Comparison of CEA between OSA and control group. Abbreviation: CEA=carcinoembryonic antigen, OSA=obstructive sleep apnea 